Bond University

Research Repository

\title{
Egong
}

The effectiveness, safety and cost-effectiveness of cytisine versus varenicline for smoking cessation in an Australian population: a study protocol for a randomized controlled noninferiority trial

Thomas, Dennis; Farrell, Michael; McRobbie, Hayden; Tutka, Piotr; Petrie, Dennis; West, Robert; Siahpush, Mohammad; Gartner, Coral; Walker, Natalie; Mendelsohn, Colin P.; Hall, Wayne; Paul, Christine; Zwar, Nicholas; Ferguson, Stuart G.; Boland, Veronica C.; Richmond, Robyn; Doran, Christopher M.; Shakeshaft, Anthony; Mattick, Richard P.; Courtney, Ryan J. Published in:

Addiction

DOI:

10.1111/add. 14541

Licence:

Other

Link to output in Bond University research repository.

Recommended citation(APA):

Thomas, D., Farrell, M., McRobbie, H., Tutka, P., Petrie, D., West, R., Siahpush, M., Gartner, C., Walker, N., Mendelsohn, C. P., Hall, W., Paul, C., Zwar, N., Ferguson, S. G., Boland, V. C., Richmond, R., Doran, C. M., Shakeshaft, A., Mattick, R. P., \& Courtney, R. J. (2019). The effectiveness, safety and cost-effectiveness of cytisine versus varenicline for smoking cessation in an Australian population: a study protocol for a randomized controlled non-inferiority trial. Addiction, 114(5), 923-933. https://doi.org/10.1111/add.14541

\footnotetext{
General rights

Copyright and moral rights for the publications made accessible in the public portal are retained by the authors and/or other copyright owners and it is a condition of accessing publications that users recognise and abide by the legal requirements associated with these rights.

For more information, or if you believe that this document breaches copyright, please contact the Bond University research repository coordinator.
} 


\section{The effectiveness, safety and cost-effectiveness of cytisine versus varenicline for smoking cessation in an Australian population: A study protocol for a randomised controlled non-inferiority trial}

Thomas $D^{1}$, Farrell $M^{1}$, McRobbie $H^{1,2}$, Tutka $P^{1,3}$, Petrie $D^{4}$, West $R^{5}$, Siahpush $M^{6}$, Gartner $C^{7}$, Walker $\mathrm{N}^{8}$, Mendelsohn $\mathrm{CP}^{9}$, Hall $\mathrm{W}^{10,11}$, Paul $\mathrm{C}^{12}$, Zwar $\mathrm{N}^{9,13}$, Ferguson $\mathrm{SG}^{14}$, Boland $\mathrm{VC}^{1}$, Richmond $\mathrm{R}^{9}$, Doran $\mathrm{CM}^{15}$, Shakeshaft $\mathrm{A}^{1}$, Mattick $\mathrm{RP}^{1}$, Courtney $\mathrm{RJ}^{1 *}$.

\section{Author affiliations}

${ }^{1}$ National Drug and Alcohol Research Centre (NDARC), University of New South Wales (UNSW), Sydney, Australia

${ }^{2}$ Wolfson Institute of Preventive Medicine, Queen Mary University of London, London, UK

${ }^{3}$ Department of Experimental and Clinical Pharmacology, Laboratory for Innovative Research in Pharmacology, University of Rzeszów, Rzeszów, Poland

${ }^{4}$ Centre for Health Economics, Monash Business School, Monash University, Melbourne, Australia

${ }^{5}$ UCL Department of Behavioural Science and Health, University College London, London, UK

${ }^{6}$ College of Public Health, University of Nebraska Medical Center, Omaha, United States

${ }^{7}$ Faculty of Medicine, School of Public Health, The University of Queensland, Brisbane, Australia

${ }^{8}$ National Institute for Health Innovation, School of Population Health, University of Auckland, Auckland, New Zealand

${ }^{9}$ School of Public Health and Community Medicine, University of New South Wales, Sydney, Australia

${ }^{10}$ Faculty of Health and Behavioural Sciences, Centre for Youth Substance Abuse Research, The University of Queensland, Brisbane, Australia

${ }^{11}$ National Addiction Centre, Kings College, London, UK

${ }^{12}$ School of Medicine and Public Health, Faculty of Health and Medicine, University of Newcastle, Newcastle, Australia

${ }^{13}$ School of Medicine, University of Wollongong, Wollongong, Australia

${ }^{14}$ School of Medicine, University of Tasmania, Hobart, Australia

${ }^{15}$ Centre for Indigenous Health Equity Research, Central Queensland University, Brisbane, Australia

*Corresponding author: Ryan Courtney, National Drug and Alcohol Research Centre, University of New South Wales, Sydney NSW 2052 Australia

This article has been accepted for publication and undergone full peer review but has not been through the copyediting, typesetting, pagination and proofreading process which may lead to differences between this version and the Version of Record. Please cite this article as doi: 10.1111/add.14541 
Email: r.courtney@unsw.edu.au

Running head: Cytisine vs varenicline for tobacco addiction

Word count: 4117

Funding: This research is funded by a National Health and Medical Research Council (NHMRC) of Australia (GNT1108318) Project Grant. The National Drug and Alcohol Research Centre at the University of New South Wales is supported by funding from the Australian Government under the Substance Misuse Prevention and Service Improvements Grants Fund. RJC is supported by a Cancer Institute New South Wales Early Career Research Fellowship (GNT14/ECF/1-46).

Competing interest: No authors have received financial support from any companies for the submitted work. HM has received honoraria for speaking at smoking cessation meetings, and attending advisory board meetings, that have been organized by Johnson \& Johnson and Pfizer. PT has undertaken consultancy for Aflofarm, a manufacturer of cytisine. RW undertakes consultancy and research for and receives travel funds and hospitality from manufacturers of smoking cessation medications. CG received paid travel and accommodation from Wellmark to present at the HIV Innovation Forum 2017, which was sponsored by an unrestricted educational grant from Gilead Sciences. NW and HM have previously received cytisine from Sopharma for the conduct of a non-inferiority trial of cytisine versus NRT. NW has previously received smoking cessation medication and matching placebo from Pfizer for the conduct of a relapse prevention trial in chronic obstructive pulmonary disease patients who smoke. NW has provided consultancy to the manufacturers of smoking cessation medications, received honoraria for speaking at a research meeting and received benefits in kind and travel support from a manufacturer of smoking cessation medications. $\mathrm{CM}$ has received funding from Pfizer Australia, GlaxoSmithKline and Johnson \& Johnson Pacific for teaching, consulting and conference expenses. NZ has received honoraria from Pfizer and GlaxoSmithKline for advice on smoking cessation education programs and conference expenses. SGF has worked as a consultant to various pharmaceutical companies on matters relating to smoking cessation and has received researcher-initiated project grant funding from Pfizer (through the GRAND initiative).

Trial registration: Australian and New Zealand Clinical Trials Registry (ACTRN12616001654448).

This article is protected by copyright. All rights reserved. 


\section{ABSTRACT}

Background and aims: Smoking cessation medications are effective but often underutilised because of costs and side effects. Cytisine is a plant-based smoking cessation medication with over 50 years of use in Central and Eastern Europe. While cytisine has been found to be well-tolerated and more effective than nicotine replacement therapy, direct comparison with varenicline have not been conducted. This study evaluates the effectiveness, safety and cost-effectiveness of cytisine compared with varenicline. Design: Two arm, parallel group, randomised, non-inferiority trial, with allocation concealment and blinded outcome assessment. Setting: Australian population-based study. Participants: Adult daily smokers $(\mathrm{N}=1266)$ interested in quitting will be recruited through advertisements and Quitline telephone-based cessation support services. Intervention and comparator: Eligible participants will be randomised (1:1 ratio) to receive either cytisine capsules (25-day supply) or varenicline tablets (12-week supply), prescribed in accordance with the manufacturer's recommended dosing regimen. The medication will be mailed to each participant's nominated residential address. All participants will also be offered standard Quitline behavioural support (up to six 10-12 minute sessions). Measurements: Assessments will be undertaken by telephone at baseline, 4- and 7-months post-randomisation. Participants will also be contacted twice (two and four weeks post-randomisation) to ascertain adverse events, treatment adherence and smoking status. The primary outcome will be self-reported 6-month continuous abstinence from smoking, verified by carbon monoxide at 7-month follow-up. We will also evaluate the relative safety and cost-effectiveness of cytisine compared with varenicline. Secondary outcomes will include self-reported continuous and 7day point prevalence abstinence and cigarette consumption at each follow-up interview. Comments: If cytisine is as effective as varenicline, its lower cost and natural plant-based composition may make it an acceptable and affordable smoking cessation medication that could save millions of lives worldwide.

Keywords Cytisine, varenicline, smoking cessation, tobacco, randomised controlled trial 


\section{INTRODUCTION}

Tobacco smoking continues to be a leading cause of preventable morbidity and mortality worldwide $(1,2)$. Helping smokers to quit is therefore a priority. Bupropion, nicotine replacement therapy (NRT) and varenicline improve long-term abstinence rates when used in quit attempts(3). Bupropion and NRT are equally effective and varenicline is superior to both bupropion and NRT (when used singly). On current evidence varenicline is the most effective sole pharmacotherapy for smoking cessation(3). The safety of varenicline is also established. A recent large-scale $(n=8144)$ study established its safety in smokers with and without psychiatric disorders (4).

Smoking cessation interventions can be highly cost-effective, but some medications come at a substantial cost to the health care sector and consumers and so may divert significant resources away from other interventions or be unaffordable(5). For example, in 2017, Australia's Pharmaceutical Benefit Scheme spent more than $\$ 30$ million subsidising varenicline(6). A wider choice of more cost-effective methods is needed to reduce the costs associated with smoking cessation and the global smoking burden in low- and middleincome countries (LMICs), where smoking cessation medications are unavailable, or cost prohibitive(7, 8).

Cytisine is a plant-based alkaloid found in members of the Leguminosae family (e.g., Cytisus laburnum)(9). Like varenicline, cytisine is a partial agonist of nicotinic acetylcholine receptors (nAChRs), binding with high affinity to the $\alpha 4 \beta 2$ subtype, which is central to the effect of nicotine on the reward pathway. Cytisine acts by reducing the rewarding effect of nicotine and attenuating nicotine withdrawal symptoms $(10,11)$.

Cytisine was first used in Bulgaria as a smoking cessation aid in the early 1960s providing inexpensive and effective cessation support to millions of smokers in Central and Eastern Europe(10). Modelling suggests cytisine may produce substantial cost savings for the global healthcare system if used instead of common smoking cessation pharmacotherapies(12). A review concluded that cytisine is the most promising future smoking cessation treatment(13).

Cytisine is: (i) inexpensive to produce $(9,14)$ and currently priced at a half to a twentieth of other cessation medications(9); (ii) well-tolerated by smokers(15); (iii) requires a shorter treatment period (i.e., 25 days) than the 56 to 84 days for NRT, bupropion and varenicline(15); and (iv) plant-based which may be attractive for smokers who prefer 'natural' medicines(16).

Nine placebo-controlled and seven uncontrolled studies have demonstrated the safety and efficacy of cytisine $(15,17)$. Most of these studies did not meet contemporary standards and were published in non-English language Journals and hence cytisine did not receive considerable interest outside of Europe until a review was published by Etter et al in 2006 $(14,17,18)$. One high-quality pragmatic non-inferiority trial $(n=1310)$ found that cytisine was superior to NRT in improving self-reported continuous abstinence rates at one week, and one, two and six months when both groups received minimal behavioural support(19). Although the self-reported adverse event $(\mathrm{AE})$ rates were higher in the cytisine group than in the NRT group ( $31 \%$ and $20 \%$ respectively), the majority of events were mild to moderate in severity and self-limited. The most frequent AEs in the cytisine arm were nausea/vomiting and sleep disorders ( $4.6 \%$ and $4.3 \%$ of the total sample that received cytisine respectively). Among smokers who received cytisine and reported an $\mathrm{AE}$, over $82 \%$ would recommend cytisine to a smoker who wanted to quit. Moreover, West et al in 2011 reported that the latest Periodic Safety Update provided to European authorities (based on seven million cytisine users) did not identify any safety concerns(20). 
Cytisine may be as effective, cheaper and therefore more cost-effective than varenicline(21). However, there are no clinical trials comparing cytisine with varenicline in the same population. The primary objective of this study is to evaluate the effectiveness of cytisine compared to varenicline on six-month continuous abstinence verified at seven-month followup. We will also compare the relative safety and the cost-effectiveness of cytisine to varenicline. We hypothesise that cytisine will be at least as effective and more cost-effective than varenicline.

\section{METHODS}

\section{Design}

Parallel group, non-inferiority, randomised controlled cost-effectiveness trial .

\section{Setting}

This is a population-based study undertaken in New South Wales, Australia. Screening and consenting processes will be completed by staff at the Trial Coordinating Centre (TCC) located at the University of New South Wales (UNSW), National Drug and Alcohol Research Centre (NDARC), Sydney, Australia. Baseline and follow-up computer assisted telephone interviews (CATIs) will be completed by an independent Contract Research Organisation (CRO) - The Social Research Centre (SRC) located in Melbourne, Australia.

\section{Study criteria}

\section{Inclusion criteria}

At least 18 years of age; current daily smoker (smoking at least one cigarette per day at the time of screening); willing to make a quit attempt using the medication provided (cytisine or varenicline); able to provide verbal informed consent; have access to a telephone; and willing to complete baseline and follow-up telephone interviews.

\section{Exclusion criteria}

Pregnant, breastfeeding or planning to become pregnant in the next 7-months; currently using smoking cessation medications; participating in another smoking cessation study; having known hypersensitivity to the active substance or to any of the excipients; hospitalised for any of the following medical conditions in the previous 3-months: arrhythmia, heart attack, stroke, severe angina; or diagnosed with pheochromocytoma or hyperthyroidism.

\section{Participant recruitment}

Participants will be recruited from two recruitment streams.

\section{Quitline}

Trained Quitline counsellors will briefly inform their clients about the study (i.e., a study that provides free support to smokers to quit) and ask if they are interested in hearing more about the study. Those interested will have their contact details passed onto the research team at the TCC. 


\section{Advertisements}

We will advertise using: i) print (i.e., newspaper, letterbox flyer drops, posters and flyers displayed at primary care centres, pharmacies, notice/bill boards, and health and government organisations); ii) radio; and iii) digital media (i.e., Facebook and Google Ads, plus other online advertisements). The advertisements will include a toll-free study number for interested smokers.

\section{Recruitment procedures}

All potential participants expressing interest in the study will be asked to choose their preferred method of receiving the participant information statement (i.e., via either hard copy mail or email or link to study website). The TCC will then contact potential participants, reiterate the study details, check eligibility including current medical conditions and medications, review self-reported medical history, and if eligible, obtain verbal informed consent. The consent will include a permission to audiotape the screening interview, providing access to screening files to a study clinician (SC) and permission to transfer contact details between various stakeholders involved in the study (see Figure 1). All randomised participants will also receive a consent form for releasing their Medicare and Pharmaceutical Benefits Scheme (PBS) claims information, though it is not a requirement for study participation.

The product monographs for cytisine and varenicline advise caution for the following conditions: psychiatric illness, ischemic heart disease, heart failure, cerebrovascular diseases, obliterating arterial diseases, diabetes mellitus (caution indicated for those who are on insulin therapy), renal and hepatic insufficiency, hypertension, active gastroesophageal reflux disorder or peptic ulcer disorder and people with history of seizures. If a cautionary condition is self-reported, the SC will consult the participant via phone before enrolling them in the study. If the current status of the condition is uncertain or further information is required, the participant may be asked for a letter of clearance from their usual doctor.

Participants' mental health status will be assessed using the short and validated Kessler Psychological Distress Scale (K6)(22). All participants with a score of 13 or above (probable serious mental illness) will be referred to the SC for further evaluation before enrolment. Suicidal ideation will be assessed using adapted items from the Australian National Survey of Mental Health and Wellbeing (Did the below experience ever happen to you? The experience is "You seriously thought about committing suicide" [A serious thought is having suicidal thoughts for a few days or making plans for it or attempting to do it. It is not a momentary or passing thought at some stage of your life]. If "yes", did that experience happen to you at any time in the past 12 months?). Those who report suicidal thoughts in the previous 12-months will be evaluated by the SC.

The SC will screen and assess all consented participants' files and provide final sign off, after which participants are referred to the CRO for a baseline CATI. Details of consented participants will be sent to the CRO using a secured file transfer system (ShareFile ${ }^{\circledR}$ ). Further information is provided in the study flow diagram (See Figure 1).

\section{Randomisation: allocation concealment and sequence generation}

Participants will be randomised to treatment after completion of the baseline CATI. The data collection system at the CRO (UNICOM ${ }^{\circledR}$ Intelligence) will assign a unique randomisation number to all participants using a pre-generated randomisation list embedded in the system. The allocations will be undertaken using the permuted block design (with unequal block 
sizes of 12 and 16)(23). After baseline interview, the system will randomise participants to either of the treatment arms in a 1:1 ratio. Only the independent statistician will have access to the pre-generated randomisation list.

\section{Blinding}

All outcome assessments will be conducted by the independent CRO. The interviewers who conduct outcome assessments will use the unique study ID (i.e., randomisation number) and not know the person's treatment allocation. To prevent participants revealing their treatment allocation to the interviewer, the person conducting follow-up assessments will use a protocol that instructs participants at the outset of each interview not to discuss treatment allocations with the interviewer. Because of differences in dosage form, dosing regimen, treatment duration and quit day, participants and research staff (who perform day-to-day activities and conduct check-in calls) cannot be blinded.

\section{Study treatments}

Participants will be randomised to receive either cytisine or varenicline. The CRO will provide the information on the allocated medication and designated quit date after randomisation. Participants will be advised to follow the dosing regimen as recommended by the manufacturer (See Supplementary Table 1 and 2). Study medications and materials will be delivered to participants' residential address by mail from a central pharmacy- ePharmacy located in Brisbane, Australia. A trained study pharmacist at the central pharmacy will reevaluate participants' medical and medication history before dispensing the study medications.

Intervention group

Participants in the intervention group will receive the standard 25-day supply of $1.5 \mathrm{mg}$ cytisine micronised capsules (Desmoxan brand). The designated quit date will be on the fifth day from the start of the treatment. The medication will be sent in one shipment.

\section{Comparator group}

Participants in the comparator group will receive the standard 12-week supply of $0.5 \mathrm{mg} / 1.0$ $\mathrm{mg}$ varenicline tablets (Champix brand). The initiation pack (4-week supply) will be dispatched immediately after randomisation. The continuation pack (8-week supply) will be dispatched to those who have started the initiation pack (as confirmed by phone) and are interested to continue treatment. The designated quit date will be on the eighth day from the start of the treatment.

\section{Quitline support}

All participants will be offered standard Quitline behavioural support. This includes a free call-back service with scheduled additional support calls (six 10-12 minutes sessions) from a counsellor, and a quit kit containing self-help quitting guides(24). TCC staff will complete an online referral form on behalf of participants who gave consent to be referred to Quitline. Consent to receive Quitline support is not required for study enrolment and those recruited via Quitline will not be referred. 


\section{DATA COLLECTION AND MEASUREMENTS}

\section{Follow-up}

The independent CRO will complete all assessments at baseline, and at 4- and 7-month post-randomisation (See Figure 2) using a structured CATI program. Participants will be reimbursed $\$ 40$ for completing each interview.

\section{Check-in calls}

All participants will receive two check-in calls (See Figure 2). The non-adherence and adverse side effects usually occur during the initial phase of treatment and hence we will conduct 2 check-in calls during the first month of treatment. This is the first study using check-in calls to capture a comprehensive data on cytisine's safety, and more than 2 checkin calls are not feasible due to study budget constraints. These calls will be conducted by trained research staff at the TCC. The study clinician will be notified of any unusual or serious health problems identified during these calls.

\section{Treatment adherence}

Participants will receive a clear instruction on the dosage regime immediately after randomisation by the $\mathrm{CRO}$. The participants will also receive consumer medicine information and a leaflet detailing dosage regimen, designated quit day, common side effects and study contact details along with the medication pack. The treatment adherence will be monitored during the check-in calls and those who are not adherent will be encouraged to use the medication as per the schedule. Self-reported treatment adherence will be assessed at follow-up 1 and 2.

\section{Measurements}

\section{Primary outcome}

The primary outcome will be biochemically verified 6-month continuous abstinence at 7month post-randomisation. Six months continuous abstinence will be defined by self-report as not having smoked more than five cigarettes for the entire 6-month period preceding the 7-month follow-up(25). Only participants who self-report continuous abstinence at 7-month post-randomisation will be biochemically verified. Participants meeting this self-reported criterion will be asked to perform a Carbon Monoxide (CO) breath test. Participants will be asked to either visit the TCC or have a trained researcher attend their home to perform this test. Participants will be reimbursed $\$ 40$ for test completion.

The $\mathrm{CO}$ level in the exhaled air will be measured using a hand-held Micro ${ }^{+}$Smokerlyzer (Bedfont Scientific Ltd, Maidstone, Kent, England). The exhaled CO level is the cheapest, easiest and most simple method of verifying tobacco use with around $90 \%$ sensitivity and specificity(26). A participant with an exhaled CO level of $\leq 9 \mathrm{ppm}$ will be considered abstinent(26). Participants who self-report abstinence but are unable or unwilling to complete $\mathrm{CO}$ test will be classified as smoking (treatment failure) for the primary outcome.

\section{Secondary outcome measures}

The secondary outcome measures are: 1) Participant self-reported continuous abstinence at each follow-up interview (3-month continuous abstinence at 4-month follow-up and 6-month continuous abstinence at 7-month follow-up; 2) Self-reported 7-day point prevalence 
abstinence (defined as prevalence of abstinence during seven consecutive days immediately preceding the follow-up) at 4- and 7-month follow-ups; and 3) Self-reported cigarette consumption at each follow-up.

\section{Safety monitoring}

Participants will complete two check-in calls and two follow-up interviews during which data will be collected on the type, severity, causality, action taken, and outcome of self-reported AEs will be collected. A toll-free number will be available for participants to self-report any $\mathrm{AE}$ and seek advice from staff at the TCC.

An AE will include any illness, sign, symptom, or clinically significant abnormality that has appeared or worsened during the clinical trial, regardless of its causal relationship to the study medications. All AEs will be carefully evaluated and summarised using MedDRA (Medical Dictionary for Regulatory Activities) coding. The severity will be assessed using Common Terminology Criteria for Adverse Events (CTCAE) version 4.0 general guidelines (i.e. grade 1 - Mild $A E$; grade 2 - moderate $A E$; grade 3 - severe $A E$; grade 4 - lifethreatening or disabling $A E$; and grade 5 - death related to $A E$ ). Causality will be assessed using the World Health Organization (WHO) criteria for causality assessment (certain, probable/likely, possible, unlikely, conditional/unclassified and unassessable/unclassifiable).

There are no formal stopping rules set for this study, but an independent Data Safety Monitoring Board (DSMB) will evaluate the safety data emerging from the study at least biannually and make recommendations on whether to continue the trial without changes, continue with changes or terminate the trial early.

\section{Other measurements}

A number of potential baseline covariates, mediating factors and other outcome variables will also be collected at baseline and at each follow-up. Table 1 lists the data collection schedule for all variables (primary, secondary, covariates, mediating factors and exploratory outcomes).

\section{Sample size}

A sample size of 1266 (633 per group) will confer $90 \%$ power at the one-sided significance level of 0.025 to detect a non-inferiority margin of $5 \%$ between the two groups at 7 -month follow-up. The 6-month self-reported continuous abstinence rate was $22 \%$ in the cytisine group in a recent pragmatic trial in a similar group of smokers, i.e. motivated Quitline callers(19). For the power calculation, we have assumed a conservative $19 \%$ biochemically verified quit rate in the cytisine group. A Cochrane review identified continuous abstinence rates ( $\geq 6$ months) for varenicline as approximately $27 \%$ compared to placebo(27). However, pragmatic evaluations have identified much lower rates of smoking cessation with varenicline and heterogeneity according to clinical practice and the population treated(28). For example, a recent study $(n=3,116)$ assessing Quitline enrolees using varenicline identified a $17 \%$ continuous cessation rate at 6 -months(29). A recent modelling study estimated that cytisine will be more clinically effective than varenicline(21). For the power calculation, we have assumed a $17 \%$ quit rate in the comparator arm given that this is a pragmatic population-based study. 


\section{Data management}

The data at the TCC will be captured in a specially designed Microsoft SQL database. The clinician and central pharmacy will have restricted access to the SQL database using a secured gateway GlobalProtect. The data collected at the CRO will be stored in an electronic database, $\mathrm{UNICOM}^{\circledR}$ Intelligence. An automatic file transfer system will be designed by connecting MS SQL database with CRO and central pharmacy databases using a secured file exchange portal, ShareFile ${ }^{\circledR}$. This will mediate the automatic file transfer between different stakeholders. The data will be encrypted in transit and in storage.

\section{Data analysis}

\section{Baseline characteristics}

Baseline characteristics of the cytisine and varenicline group will be presented using frequency and percentages for categorical variables and means/standard deviations for continuous measures.

\section{Treatment effects}

The analysis plan will be pre-specified. The primary analysis will compare the quit rates between the two groups. Regression analysis will be used to compare quit rates between the two treatment groups (both adjusted and unadjusted). Non-inferiority will be evaluated by observing whether the lower bound of the 95\% confidence interval for the risk difference in quit rate between cytisine and varenicline is above the non-inferiority margin $(-5 \%)$.

The primary analysis will be conducted according to intention-to-treat (ITT) principles where missing data is treated as smokers. Sensitivity analyses will involve: (1) using multiple imputation to account for missing data; (2) excluding participants with missing data; and (3) excluding participants with protocol deviations (e.g., not adherent to treatment)(30).

Similar analyses (logistic regression) will be undertaken for the secondary cessation outcomes at each follow-up point. There will be an additional combined analysis of all time points using a regression model that includes time as a variable and uses a random intercept to adjust for correlated observations among individuals over time. The consistency of effects among the following subgroups will also be assessed using tests for heterogeneity (random-effects model) for primary outcome: age, gender, nicotine dependence (assessed using 2-item Heaviness of Smoking Index $(\mathrm{HSI}) \geq 4$ will be considered as heavy smokers and an $\mathrm{HSI}$ score of $\leq 3$ will be considered as light smokers) and presence of any mental health condition. Regression models which adjust for mediating factors such as treatment adherence and Quitline use will also be analysed.

\section{Health economic analyses}

The cost-effectiveness analysis will take a healthcare system perspective. Costs will be captured by staff recording the time taken and resources used for assessment, administration, drug related costs and treating any complications. Downstream costs associated with government subsidised primary care use and other drug use will be collected by linkage with Medicare records. First the following within-trial analyses will be undertaken. The incremental costs between the cytisine and varenicline groups and incremental costs per additional quitter after the 7 months follow-up will be estimated. In addition, we will estimate the cost-effectiveness in terms of the incremental cost per additional Quality Adjusted Life Year (QALY) - as measured by linear interpolation between baseline, follow-up 1 and follow-up 2 QALYs weights as captured by the EQ-5D-5L. 
Given that the majority of the health benefits and cost savings from quitting are likely to be experienced after the trial, the implications for lifetime QALYs and future costs will be modelled using the same Markov model structure as in the recent UK HTA report(21) to estimate the cost per QALY. Relapse rates and other parameters in the model will be estimated using the Household Income Labour Dynamics in Australia (HILDA) survey data, a large annual longitudinal cohort which began in 2001. Healthcare cost information will be used from the Australian Institute of Health and Welfare (AlHW) to estimate the savings from reduced morbidity.

All analyses will involve probabilistic sensitivity analysis to explore the robustness of the conclusions and explore the effects of lower levels of patient adherence. Our estimated incremental cost effectiveness ratios will also be compared with those estimated in the literature simply based on effectiveness of the intervention, the cost of the intervention and other standardised inputs(12). Given that cytisine is not available in Australia, a threshold analysis will determine the maximum price the government should be willing to pay. Planned sub-group analyses will also be conducted including nicotine dependence (heavy vs light smokers), presence of any mental health condition and socioeconomic status.

\section{Tolerability analysis}

All randomised participants who take at least one dose of cytisine or varenicline will be included in the safety analyses. The incidence of $A E$ and frequency of treatment discontinuation in both groups will be compared using Chi-square statistic. All suspected unexpected serious adverse events reported during the study, and meeting the following criteria, will be evaluated promptly and summarised by treatment group: results in death; lifethreatening; requires inpatient hospitalisation or prolongation of existing hospitalization; results in persistent or significant disability/incapacity; congenital abnormality/birth defect; or an important medical event.

\section{Ethics}

The trial will be conducted in compliance with the protocol, the principles of Good Clinical Practice(31), the National Health and Medical Research Council's (NHMRC) National Statement on Ethical Conduct in Human Research (2007)(32) and the Australian Code for the Responsible Conduct of Research (2007)(33). Ethical approval for this study was obtained from the University of New South Wales Human Research Ethics Committee. A Therapeutic Goods Administration (TGA) notification has been filed as cytisine is not a registered product listed on the Australian Register of Therapeutic Goods (ARTG). 


\section{DISCUSSION}

If cytisine is at least as effective as varenicline it could provide a more affordable cessation treatment option for smokers globally that would make substantial savings for health care systems and consumers. Although the smoking rate in Australia has been substantially reduced over the last two decades, $12.8 \%$ of the Australian population still smoke daily(34). The shorter treatment regimen and natural composition of cytisine may attract more smokers using it as a quitting aid leading to further decrease rates of smoking. The study has the potential to inform policy on a treatment that could annually save millions of lives worldwide, particularly in LMICs where smoking rates are rising, and existing cessation treatments are prohibitively expensive.

This study has a large sample size and rigorous study design. The telephone-based interviews and assessments (telehealth) will improve the recruitment and retention rate as it will allow greater flexibility for participants to attend interviews (no travel included)(35). All outcome measures at each follow-up interview will be collected by a CRO independent of the research team. This is the first clinical trial using cytisine micronised capsules, all the previous clinical trials of cytisine used tablets but both contain $1.5 \mathrm{mg}$ of cytisine.

This study has some limitations. Self-reported data will be used for adherence, safety and all other variables except for primary endpoint which will be biochemically verified. Since all data are collected remotely by telephone, it is difficult to accurately quantify and grade AEs (e.g., laboratory abnormalities, hypertension etc.). However, our trained research staff will prompt each participant during each interview to report any changes in their health status after commencing the study medication. Detailed information will be obtained to process each AE. All data will be collected using a structured CATI. While there are substantial differences between both arms - treatment duration (25 days versus 12 weeks), quit day $\left(5^{\text {th }}\right.$ day versus $8^{\text {th }}$ day) and dispensing practices (one versus two dispensing) - we are adhering to the manufacturers standard treatment regimen which previous clinical trials have found to be safe and effective. The existing patent of varenicline will expire in 2020 which may reduce its current price.

\section{Clinical trial registration}

Australian and New Zealand Clinical Trials Registry (ACTRN12616001654448).

\section{Current status}

The trial started recruitment in January 2018, with recruitment expected to take 18 months. Trial findings are expected to be available early 2020 .

\section{ACKNOWLEDGEMENTS}

We would like to thank all our stakeholders the SRC conducting baseline and follow-up interviews (CRO), Chemist Warehouse ePharmacy storing and dispensing medications (Central Pharmacy), Aflofarm (cytisine capsules purchased from Aflofarm), Dotsquares (database development company), and Dr Josephine Pang (our study clinician). We would also like to thank the DSMB members: Dr Julia Lappin, Dr Jason Grebely and Dr Daniel Barker. We would also like to thank Reshika Chand, Billie Townsend and Saki Talukder for their work on the project. 


\section{REFERENCES}

1. Centers for Disease Control and Prevention. Smoking \& Tobacco use. Fast Facts. 2015.

2. Peacock A, Leung J, Larney S, Colledge S, Hickman M, Rehm J, et al. Global statistics on alcohol, tobacco and illicit drug use: 2017 status report. Addiction 2018;113 (10): 1905-26.

3. Cahill K, Stevens S, Perera R, Lancaster T. Pharmacological interventions for smoking cessation: an overview and network meta-analysis. Cochrane Database Syst Rev 2013(5):CD009329.

4. Anthenelli RM, Benowitz NL, West R, St Aubin L, McRae T, Lawrence D, et al.

Neuropsychiatric safety and efficacy of varenicline, bupropion, and nicotine patch in smokers with and without psychiatric disorders (EAGLES): a double-blind, randomised, placebo-controlled clinical trial. The Lancet 2016: 387 (10037): 2507-20.

5. West R, Raw M, McNeill A, Stead L, Aveyard P, Bitton J, et al. Health-care interventions to promote and assist tobacco cessation: a review of efficacy, effectiveness and affordability for use in national guideline development. Addiction 2015;110(9):1388-403.

6. Australian Government. Department of Human Services. Pharmaceutical Benefits Schedule Item Reports 2015.

7. Barendregt JJ, Bonneux L, van der Maas PJ. The health care costs of smoking. N Engl J Med 1997;337(15):1052-7.

8. World Health Organisation. Reducing risks and preventing disease: population-wide interventions. 2011.

9. Prochaska JJ, Das S, Benowitz NL. Cytisine, the world's oldest smoking cessation aid. BMJ 2013;347:f5198.

10. Tutka P, Zatonski W. Cytisine for the treatment of nicotine addiction: from a molecule to therapeutic efficacy. Pharmacol Rep 2006;58(6):777-98.

11. Zatonski W, Cedzynska M, Tutka P, West R. An uncontrolled trial of cytisine (Tabex) for smoking cessation. Tob Control 2006;15(6):481-4.

12. Stapleton JA, West R. A direct method and ICER tables for the estimation of the costeffectiveness of smoking cessation interventions in general populations: application to a new cytisine trial and other examples. Nicotine Tob Res 2012;14(4):463-71.

13. Beard E, Shahab L, Cummings DM, Michie S, West R. New Pharmacological Agents to Aid Smoking Cessation and Tobacco Harm Reduction: What Has Been Investigated, and What Is in the Pipeline? CNS drugs 2016;30(10):1-33.

14. Aveyard $\mathrm{P}$, West R. Cytisine and the failure to market and regulate for human health. Thorax 2013;68(11):989.

15. Hajek $P$, McRobbie $H$, Myers K. Efficacy of cytisine in helping smokers quit: systematic review and meta-analysis. Thorax 2013;68(11):1037-42.

16. Thompson-Evans TP, Glover MP, Walker N. Cytisine's Potential to Be Used as a Traditional Healing Method to Help Indigenous People Stop Smoking: A Qualitative Study With Māori. Nicotine Tob Res 2011;13(5):353-60.

17. Etter JF. Cytisine for smoking cessation: a literature review and a meta-analysis. Arch Intern Med 2006;166(15):1553-9.

18. Rigotti NA. Cytisine-a tobacco treatment hiding in plain sight. N Engl J Med 2014;371:242930.

19. Walker N, Howe C, Glover M, McRobbie H, Barnes J, Nosa V, et al. Cytisine versus nicotine for smoking cessation. N Engl J Med 2014;371(25):2353-62.

20. West R, Zatonski W, Cedzynska M, Lewandowska D, Pazik J, Aveyard P, et al. Placebocontrolled trial of cytisine for smoking cessation. N Engl J Med 2011;365(13):1193-200.

21. Leaviss J, Sullivan W, Ren S, Everson-Hock E, Stevenson M, Stevens JW, et al. What is the clinical effectiveness and cost-effectiveness of cytisine compared with varenicline for smoking cessation? A systematic review and economic evaluation. Health Technol Assess 2014;18(33):1-120. 22. Kessler RC, Barker PR, Colpe LJ, Epstein JF, Gfroerer JC, Hiripi E, et al. Screening for serious mental illness in the general population. Arch of Gen Psychiatry 2003;60(2):184-9.

23. Schulz KF, Grimes DA. Unequal group sizes in randomised trials: guarding against guessing. The Lancet 2002;359(9310):966-70.

24. iCanQuit. Available at: https://www.icanquit.com.au/ (accessed 10 April 2018).

25. West R, Hajek P, Stead L, Stapleton J. Outcome criteria in smoking cessation trials: proposal for a common standard. Addiction 2005;100(3):299-303.

26. Benowitz NL, Jacob III P, Ahijevych K, Jarvis MJ, Hall S, LeHouezec J, et al. Biochemical verification of tobacco use and cessation. Nicotine Tob Res 2002;4(2). 
27. Cahill K, Lindson-Hawley N, Thomas KH, Fanshawe TR, Lancaster T. Nicotine receptor partial agonists for smoking cessation. Cochrane Database Syst Rev 2016(5):CD006103.

28. Brose LS, West R, Stapleton JA. Comparison of the effectiveness of varenicline and combination nicotine replacement therapy for smoking cessation in clinical practice. Mayo Clinic Proc 2013;88(3):226-33.

29. Biazzo LL, Froshaug DB, Harwell TS, Beck HN, Haugland C, Campbell SL, et al.

Characteristics and abstinence outcomes among tobacco quitline enrollees using varenicline or nicotine replacement therapy. Nicotine Tob Res 2010;12(6):567-73.

30. White IR, Horton NJ, Carpenter J, Pocock SJ. Strategy for intention to treat analysis in randomised trials with missing outcome data. BMJ 2011;342:d40.

31. International Conference on Harmonisation of technical requirements for registration of pharmaceuticals for human use: Good Clinical Practice. Available at: http://ichgcp.net (accessed 10 April 2018).

32. National Health and Medical Research Council: National Statement on Ethical Conduct in Human Research 2007 - updated 2009. Available at:

http://www.nhmrc.gov.au/guidelines/publications/e72 (accessed 10 April 2018).

33. National Health and Medical Research Council: Australian Code for the Responsible Conduct of Research. Available at: http://www.nhmrc.gov.au/guidelines/publications/r39 (accessed 10 April 2018)

34. Australian Institute of Health and Welfare. National Drug Strategy Household Survey detailed report 2016. Canberra: AlHW; 2017.

35. Courtney RJ, Clare P, Boland V, Martire KA, Bonevski B, Hall W, et al. Predictors of retention in a randomised trial of smoking cessation in low-socioeconomic status Australian smokers. Addict Behav 2017;64:13-20.

36. Borland $\mathrm{R}$, Yong $\mathrm{H}-\mathrm{H}$, O'connor $\mathrm{R}$, Hyland $\mathrm{A}$, Thompson $\mathrm{M}$. The reliability and predictive validity of the Heaviness of Smoking Index and its two components: findings from the International Tobacco Control Four Country study. Nicotine Tob Res 2010;12:S45-S50.

37. West R, Hajek P. Evaluation of the mood and physical symptoms scale (MPSS) to assess cigarette withdrawal. Psychopharmacology 2004;177(1-2):195-9.

38. Bush K, Kivlahan DR, McDonell MB, Fihn SD, Bradley KA. The AUDIT alcohol consumption questions (AUDIT-C): an effective brief screening test for problem drinking. Arch Intern Med 1998;158(16):1789-95.

39. Herdman M, Gudex C, Lloyd A, Janssen M, Kind P, Parkin D, et al. Development and preliminary testing of the new five-level version of EQ-5D (EQ-5D-5L). Qual Life Res $2011 ; 20(10): 1727-36$.

40. Lovibond S. H., Lovibond P. F. Manual for the Depression Anxiety Stress Scales, 2nd edn. Sydney: Psychology Foundation; 1995.

41. Siahpush M, Carlin JB. Financial stress, smoking cessation and relapse: results from a prospective study of an Australian national sample. Addiction 2006;101(1):121-7.

This article is protected by copyright. All rights reserved. 


\section{Recruitment}

- Advertisements: Interested smokers will call TCC; TCC staff will briefly provide study information and send PIS

- Quitline referrals: Counsellors based at Quitline service will inform callers about the study and ask whether they are interested in hearing about a study offering free support to quit; Interested smokers will be referred to the TCC and sent PIS

$\downarrow$

Screening and consenting - TCC (within 1-3 days after sending PIS)

Provide further study information \& answer any queries

- Assess eligibility criteria, health conditions and obtain verbal consent

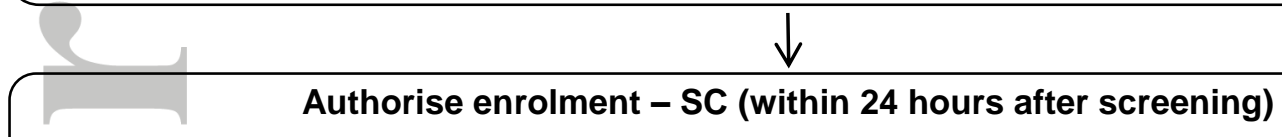

- SC to screen participant files, check audio recordings, complete teleconsultation (as required), and provide final approval for study enrolment

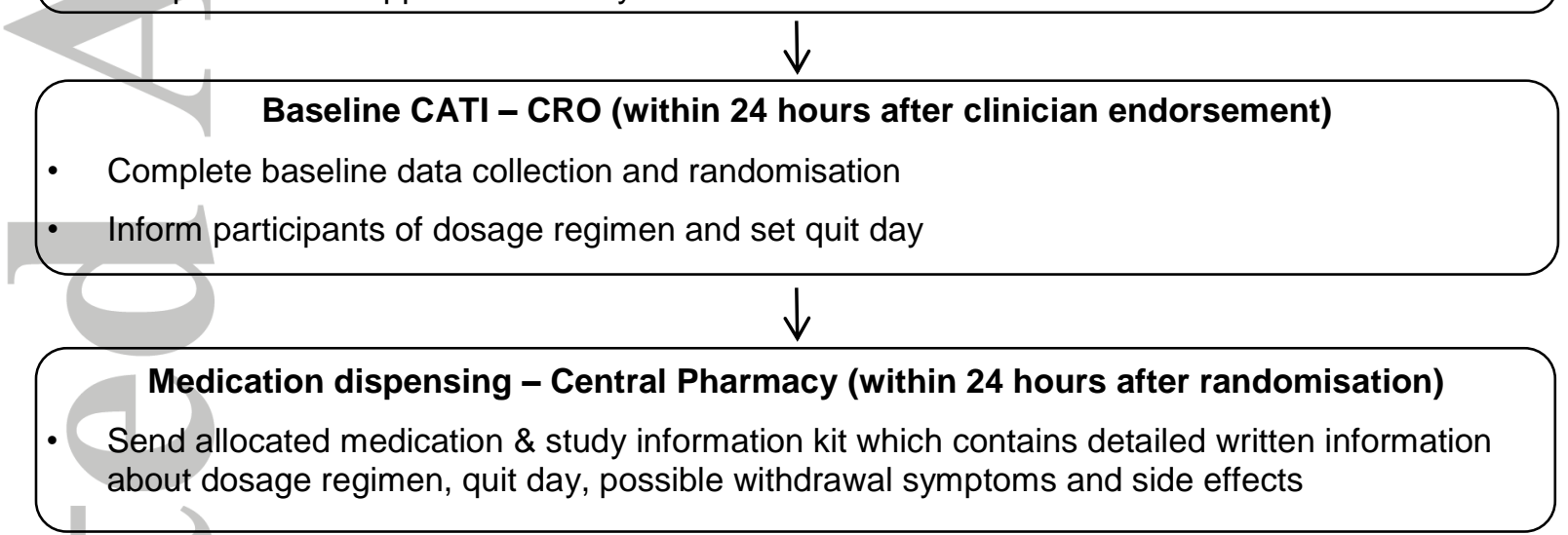

$\downarrow$
Check-in calls - TCC
Two check-in calls (at 2 weeks and 4 weeks from randomisation) to check safety, adherence
and smoking status

\[ \]
Follow-up CATIs - CRO
Follow-up 1: 4 months after randomisation
Follow-up 2: 7 months after randomisation

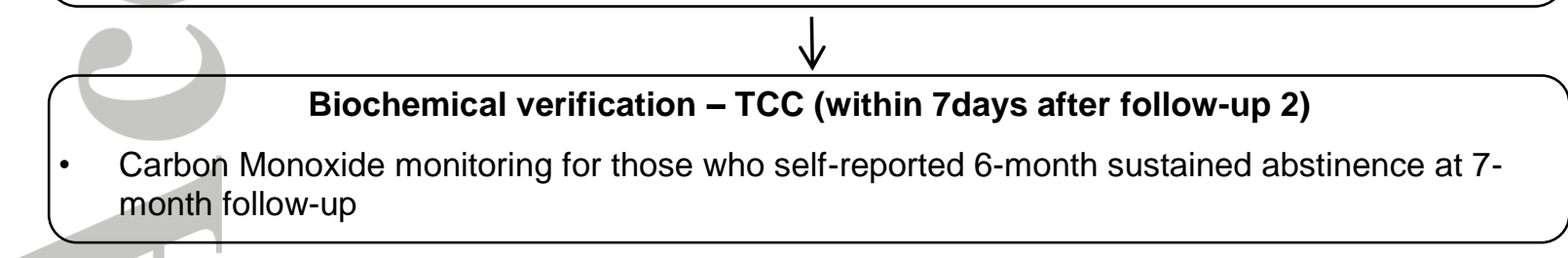

Abbreviations: TCC, Trial Coordinating Centre; PIS, Participant Information Sheet; SC, Study Clinician; CATI, Computer Assisted Telephone Interview; CRO, Contract Research Organisation

This article is protected by copyright. All rights reserved. 
Figure 2: Study Plan Schematic

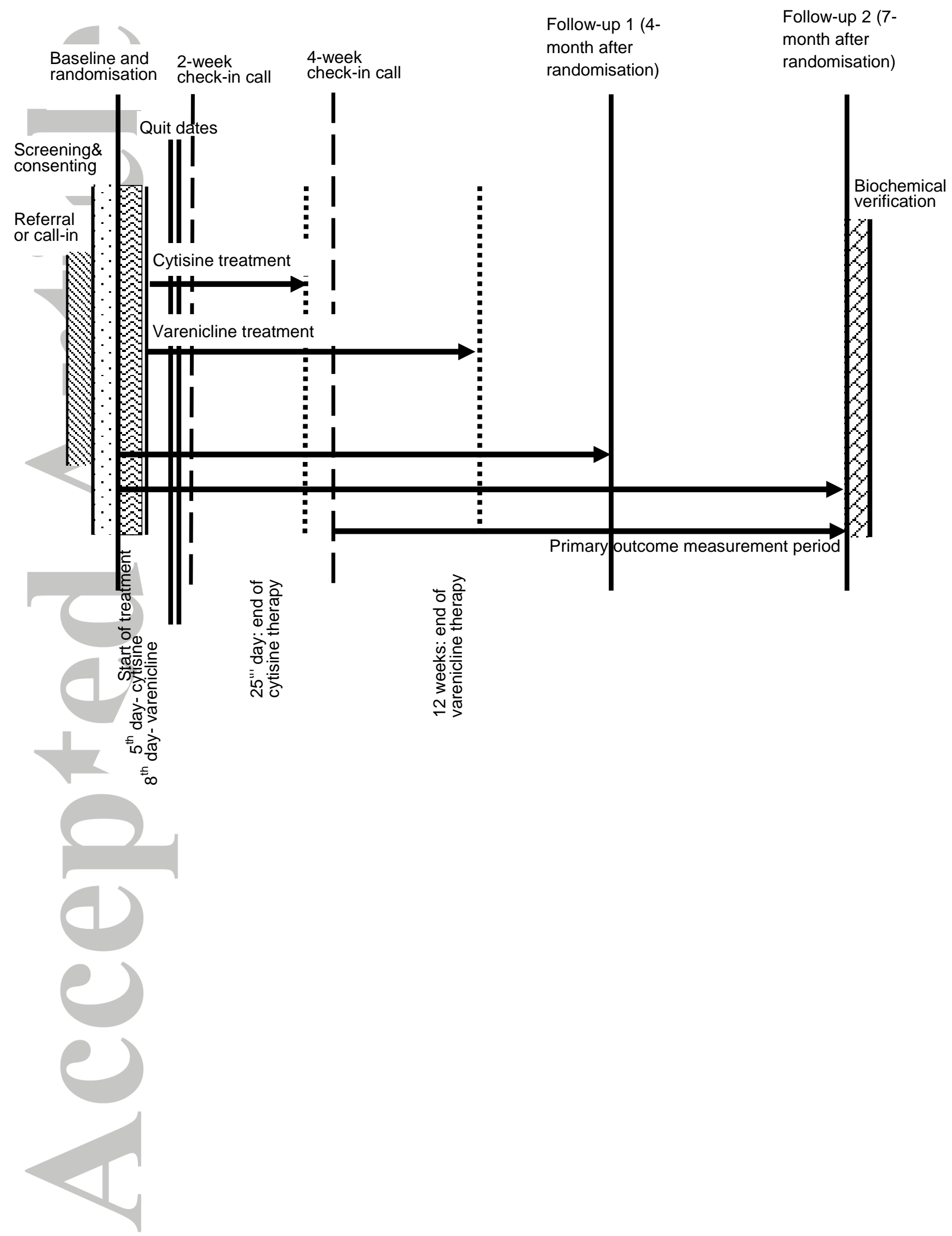


Table 1: Summary of assessments

\begin{tabular}{|c|c|c|c|c|c|}
\hline Variable & $\begin{array}{l}\text { Scree } \\
\text { ning }\end{array}$ & $\begin{array}{l}\text { Basel } \\
\text { ine }\end{array}$ & $\begin{array}{l}\text { Chec } \\
\text { k-in } \\
\text { calls }\end{array}$ & $\begin{array}{l}\text { Follow- } \\
\text { up } 1 \text { (4 } \\
\text { months) }\end{array}$ & $\begin{array}{l}\text { Follow- } \\
\text { up } 2 \text { ( } 7 \\
\text { months) }\end{array}$ \\
\hline Age, sex, medical history review and eligibility screening & $\sqrt{ }$ & & & & \\
\hline $\begin{array}{l}\text { Socio-demographic variables: Country of birth, Aboriginal or } \\
\text { Torres Strait Islander status, education, employment status, } \\
\text { marital status, current living arrangement, average annual } \\
\text { household gross income and possession of any concession } \\
\text { card. }\end{array}$ & & $\sqrt{ }$ & & & \\
\hline Current medications and medical conditions & $\sqrt{ }$ & & $\sqrt{ }$ & $\sqrt{ }$ & $\sqrt{ }$ \\
\hline Smoking status and number of cigarettes smoked/day & & $\sqrt{ }$ & $\sqrt{ }$ & $\sqrt{ }$ & $\sqrt{ }$ \\
\hline Pregnancy & $\sqrt{ }$ & & & $\sqrt{ }$ & $\sqrt{ }$ \\
\hline Access to healthcare services & & $\sqrt{ }$ & & & \\
\hline $\begin{array}{l}\text { Smoking habit: Age started; number of cigarettes smoked } \\
\text { per day; previous quitting experiences and methods used; } \\
\text { use and experiences with Quitline, NRT, prescription } \\
\text { medications or electronic cigarettes; perceptions about } \\
\text { smoking cessation advertisements and taxation; smoking } \\
\text { cessation care received from health professionals; type of } \\
\text { cigarettes smoked (e.g. roll-your-own and/or factory-made); } \\
\text { and money spent on cigarette/week. }\end{array}$ & & $\sqrt{ }$ & & & \\
\hline $\begin{array}{l}\text { Nicotine dependence: The nicotine dependence will be } \\
\text { assessed using 2-item Heaviness of Smoking Index } \\
(\mathrm{HSI})(36) \text {. }\end{array}$ & & $\sqrt{ }$ & & $\sqrt{ }$ & $\sqrt{ }$ \\
\hline $\begin{array}{l}\text { Intention to quit smoking assessed using } 4 \text { point criteria (will } \\
\text { quit in the next } 30 \text { days; will quit in the next } 6 \text { months, but not } \\
\text { in the next } 30 \text { days; may quit in the future, but not in the next } \\
6 \text { months; never expect to quit). }\end{array}$ & & & & $\sqrt{ }$ & $\sqrt{ }$ \\
\hline $\begin{array}{l}\text { The physical signs and symptoms associated with } \\
\text { withdrawal: This will be measured using } 12 \text {-item Mood and } \\
\text { Physical Symptoms Scale (MPSS)(37). }\end{array}$ & & $\sqrt{ }$ & $\sqrt{ }$ & $\sqrt{ }$ & $\sqrt{ }$ \\
\hline $\begin{array}{l}\text { Alcohol use and abuse: This will be assessed using 3-item } \\
\text { Alcohol Use Disorders Identification Test (AUDIT-C)(38). }\end{array}$ & & $\sqrt{ }$ & $\sqrt{ }$ & $\sqrt{ }$ & $\sqrt{ }$ \\
\hline Cannabis use in the last month & & $\sqrt{ }$ & & $\sqrt{ }$ & $\sqrt{ }$ \\
\hline $\begin{array}{l}\text { Health related quality of life: This will be measured using } \\
\text { EuroQol five dimension questionnaire (EQ-5D-5L)(39). }\end{array}$ & & $\sqrt{ }$ & & $\sqrt{ }$ & $\sqrt{ }$ \\
\hline $\begin{array}{l}\text { Mental distress using } 21 \text { item Depression Anxiety Stress } \\
\text { Scales (DASS)(40). }\end{array}$ & & $\sqrt{ }$ & & $\sqrt{ }$ & $\sqrt{ }$ \\
\hline
\end{tabular}

This article is protected by copyright. All rights reserved. 


\begin{tabular}{|l|l|l|l|l|l|}
\hline Financial stress using Index of Financial Stress(41). & & $\sqrt{ }$ & & $\sqrt{ }$ & $\sqrt{ }$ \\
\hline Use of other medications and other methods of cessation & & & & $\sqrt{ }$ & $\sqrt{ }$ \\
\hline $\begin{array}{l}\text { Treatment adherence: The self-reported medications use } \\
\text { (i.e., number of capsules/tablets used) }\end{array}$ & & & $\sqrt{ }$ & $\sqrt{ }$ & \\
\hline Safety & & & $\sqrt{ }$ & $\sqrt{ }$ & $\sqrt{ }$ \\
\hline Other health resource use & & & & $\sqrt{ }$ & $\sqrt{ }$ \\
\hline
\end{tabular}
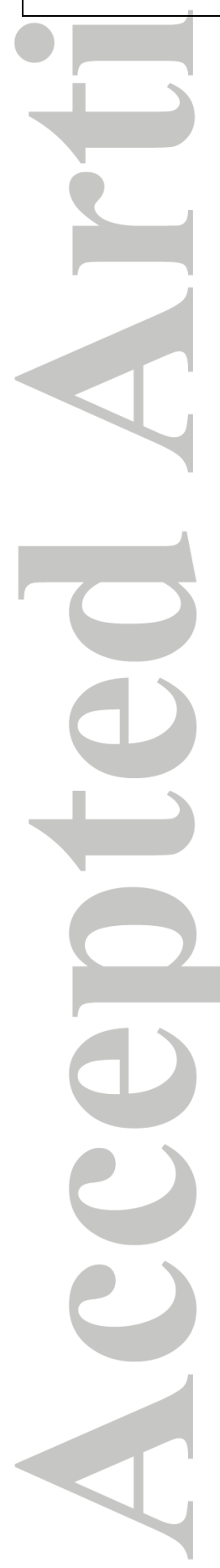

This article is protected by copyright. All rights reserved. 